\title{
Distinct processing of function verb categories in the human brain
}

\author{
Daniela Briem ${ }^{a}$, Britta Balliel ${ }^{a}$, Brigitte Rockstroh ${ }^{a, *}$, Miriam Butt $^{b}$, \\ Sabine Schulte im Walde ${ }^{c}$, Ramin Assadollahi ${ }^{a, d}$
}

${ }^{a}$ University of Konstanz, Department of Psychology, Germany

bUniversity of Konstanz, Department of Linguistics, Germany

${ }^{\mathrm{C}}$ University of Stuttgart, Institute for Natural Language Processing, Germany

${ }^{\mathrm{d}}$ ExB Communication Systems, Munich, Germany

\section{A R T I C L E I N F O}

Article history:

Accepted 9 October 2008

Available online 28 October 2008

Keywords:

Magnetencephalography

MEG

Function verb

Light verb

Context

Underspecification

Thematic relation

Complex predicate

\begin{abstract}
A B S T R A C T
A subset of German function verbs can be used either in a full, concrete, 'heavy' ("take a computer") or in a more metaphorical, abstract or 'light' meaning ("take a shower", no actual 'taking' involved). The present magnetencephalographic (MEG) study explored whether this subset of 'light' verbs is represented in distinct cortical processes. A random sequence of German 'heavy', 'light', and pseudo verbs was visually presented in three runs to 22 native German speakers, who performed lexical decision task on real versus pseudo verbs. Across runs, verbs were presented (a) in isolation, (b) in minimal context of a personal pronoun, and (c) 'light' verbs only in a disambiguating context sentence. Central posterior activity 95$135 \mathrm{~ms}$ after stimulus onset was more pronounced for 'heavy' than for 'light' uses, whether presented in isolation or in minimal context. Minimal context produced a similar heavy > light differentiation in the left visual word form area at 160-200 ms. 'Light' verbs presented in sentence context allowing only for a 'heavy reading' evoked larger lefttemporal activation around $270-340 \mathrm{~ms}$ than the corresponding 'light reading'. Across runs, real verbs provoked more pronounced activation than pseudo verbs in left-occipital regions at 110-150 ms. Thus, 'heavy' versus 'light readings' of verbs already modulate early posterior visual evoked response even when verbs are presented in isolation. This response becomes clearer in the disambiguating contextual condition. This type of study shows for the first time that language processing is sensitive to representational differences between two readings of one and the same verb stem.
\end{abstract}

\section{Introduction}

Understanding language involves the retrieval of meaning from what we hear or read. Linguistic and neurolinguistic research has investigated how meaning is carried by the syntax and the semantics of words. Verbs are of particular interest, as they directly determine the overall form and meaning of the sentence. Brain activity evoked by verbs has been one route to understanding how the brain retrieves meaning. Studies have demonstrated that the processing of word (or verb) categories that are distinguished by features such as number of arguments (Assadollahi and Rockstroh,

\footnotetext{
* Corresponding author. FB Psychologie, PO Box D23, Universität Konstanz, D-78457 Konstanz, Germany. Fax: +49 7531882891.

E-mail address: Brigitte.Rockstroh@uni-konstanz.de (B. Rockstroh).
} 
2008), frequency (Assadollahi and Pulvermüller, 2003), or association with movement (Pulvermüller et al., 2001) is reflected in distinct cortical response patterns.

The present study extends this research with an emphasis on German 'light' verbs: crosslinguistically, a subset of the verbs of a language can also be used in a 'light' way. Verbs like "take" or "give" can describe a situation, in which a physical entity is taken from or given to some person. The same verbs can also be used in sentences such as "take a shower", "give the soup a stir", or "take/give a break", where "shower/stir" or "break" are not physically being taken away or given to somebody (Other exemplars of 'light' verbs are "go", "come", "rise", "fall", with "fall into a pit" implying an actual physical falling, whereas "fall asleep" does not imply that the person asleep falls anywhere.). Thus, 'light' verbs have one morphological form but two meanings (Butt and Lahiri, 2007).

The precise representation for 'light' versus 'heavy' meanings can be understood in one of two ways: the 'light' version somehow has less information in it (hence 'light'; Butt, 2003; Butt and Geuder, 2001; Grimshaw and Mester, 1988; see also Heringer, 1968; Winhart, 2002). There is just one underlying underspecified representation which gives rise to both, with 'light' verbs being more flexible and allowing for a wider set of uses (Butt, 2003; Butt and Lahiri, 2007; Butt and Tantos, 2004). Underspecification means that 'light' verbs in a specific context do not assign the full array of thematic roles, that is, they do not determine the number, types, and placements of arguments in the same way as 'heavy' verbs in simple sentences do (Butt et al., 2008). This is because 'light' verbs need to combine with another element to form the final predication of the sentence (i.e., "phone-do" = "telephone somebody", "to give a kiss" = "to kiss").

With respect to German, the nature of the noun combined with the verb defines the main difference between its 'light' and 'heavy' meaning. Consider the examples "Paul setzt Paula unter Druck" ("Paul puts Paula under pressure") versus "Paul setzt Paula auf den Stuhl" ("Paul seats Paula on a chair"). The former illustrates the verb "setzen" ("to seat, place") in a "light reading', the latter in a full ('heavy') reading. In the latter, "Paul" is clearly the agent of the action, "Paula" is the theme/ patient of the action and "the chair" represents a concrete location. In the 'light reading', on the other hand, while "Paul" is still the agent and "Paula" the theme, it is not really clear what "Druck" ("the pressure") is. This is because of the combination of the noun with the 'light' use of the verb "setzen" ("to seat, place") in order to express a verbal predication meaning "drücken" ("to pressure").

The nature of the specific noun combined with a verb thus defines the main difference between its 'light' and 'heavy' meaning. In the case of "unter Druck", the noun is derived from a 'heavy' verb "drücken" ("to push"), and is a so called 'verbal noun', which retains predicational ability of the verbal form. As such, the verbal noun can contribute its own argument structure to the sentence. Consider the example sentence "Paul bringt Paula mit Geld in Verbindung" ("Paul connects Paula with money"; lit. "Paul brings Paula into connection with money"). Here, in German, "Verbindung" ("connection") is derived from the verb "verbinden" ("to tie together"), which requires an agent ("Paul"), a theme/patient ("Paula") and something with which this theme/patient is connected ("Geld"; engl. "money"). The verb "bringen" ("to bring") itself only requires an agent and a theme, e.g., "Paul bringt Paula Schokolade" ("Paul brings Paula chocolate"). The combination of "Verbindung" and "bringen" thus results in a combined argument structure. Both parts of the predication license the agent ("Paul") and the theme/patient ("Paula"), but only "Verbindung" licences the argument "mit Geld" ("with money"). That is, we have an instance of a joint complex predication, which is the result so-called argument-merging, in which there are at least two predicates contributing structural information to one single event (Butt, 1995, 2003).

As stated above, verbs bear information about thematic roles: 'Heavy' verbs comprise a full array of information about semantic argument structure. 'Light' verbs in contrast are assumed to have a variable in their argument structure that calls for another predicate to be substituted in (Butt et al., 2008). This property of verbs - to assign thematic roles - is supposed to be an important reason for determining the overall form and meaning of the sentence because thematic roles disclose the relations of the participants involved in the described event. And in that, thematic roles can mediate between syntax and semantics. That is, the concept of thematic roles is related to grammatical functions (subject, object) and those, again, are mapped to expressions of case (nominative, accusative, etc.). This so-called form-to-meaning mapping what is captured e.g. in the 'Linking theory' within 'Lexical functional grammar' (Bresnan and Zaenen, 1990; Butt and Dalrymple, 1997;

Fig. 1 - Top: Grand mean of MNE for the left hemisphere, averaged separately for the three conditions or runs (run 1, verbs in isolation: solid lines; run 2, verbs in minimal context: dashed lines; run 3, 'light' verbs in sentence context: dotted lines) across stimuli (verb categories). Circles mark activity peaks, which were chosen for further analyses; abscissa: time scale in ms after verb onset, vertical scale marks in steps of $100 \mathrm{~ms}$; ordinate: activity in $\mathrm{nAm} / \mathrm{cm}^{2}$. Mid: Topographical distribution of cortical activation (left-hemispheric view) in the source space (MNE) projected onto a smoothed standard brain (Junghöfer and Peyk, 2004). Color shades indicate differences in activation between verb categories (from +3 to $-3 \mathrm{nAm} / \mathrm{cm}^{2}$ ); red color: larger activation by 'heavy' than by 'light' verbs in runs 1 and 2, respective by 'light' verbs in 'heavy' relative to 'light reading' in run 3 ; blue: reverse difference. Arrows mark the areas of regions of interest selected for statistical evaluation of verb category differences. A: Topographical distribution of activation evoked by verbs in isolation in run 1 for the time interval 95-135 ms after verb onset; B: Topographical distribution of activation evoked by verbs in minimal context in run 2 for the time interval 160-200 ms after verb onset; C: Topographical distribution of activation evoked by 'light' verbs in 'heavy' or 'light reading' in run 3 in the time interval 270-340 ms after verb onset. Bottom: Grand mean of MNE for the selected regions of interest with significant activity differences between verb categories for the particular time segment. Abscissa: time scale in msec after verb onset, vertical scale marks in steps of $100 \mathrm{~ms}$, grey shaded bar indicates selected time window; ordinate: activity in $\mathrm{nAm} / \mathrm{cm}^{2}$. Time window in $\mathrm{ms}$, ordinate: activity in $\mathrm{nAm} / \mathrm{cm}^{2}$. 
Dalrymple, 2001; Higginbotham, 1983) therefore plays a mayor role in all domains of linguistic explanations.

Under the assumption that thematic roles are mapped to grammatical functions, in complex predicates including underspecified ('light') verbs, the corresponding of the lexical semantics of a verb (thematic roles), the grammatical functions (subject, object) and the expression of case (nominative, accusative, etc.) should differ from those of specified 'heavy' verb sentences.
Following previous studies, which indicate distinct brain processing of distinct verb categories (e.g., Assadollahi and Rockstroh, 2008), the present study explored whether verbs that could be used with both 'light' and 'heavy' meaning would be processed differently in the brain compared to verbs with an unambiguous 'heavy' meaning. In addition, the study explored whether and how a disambiguating sentence context, which disambiguated towards either a 'light' or a 'heavy reading' of the verb would result in a
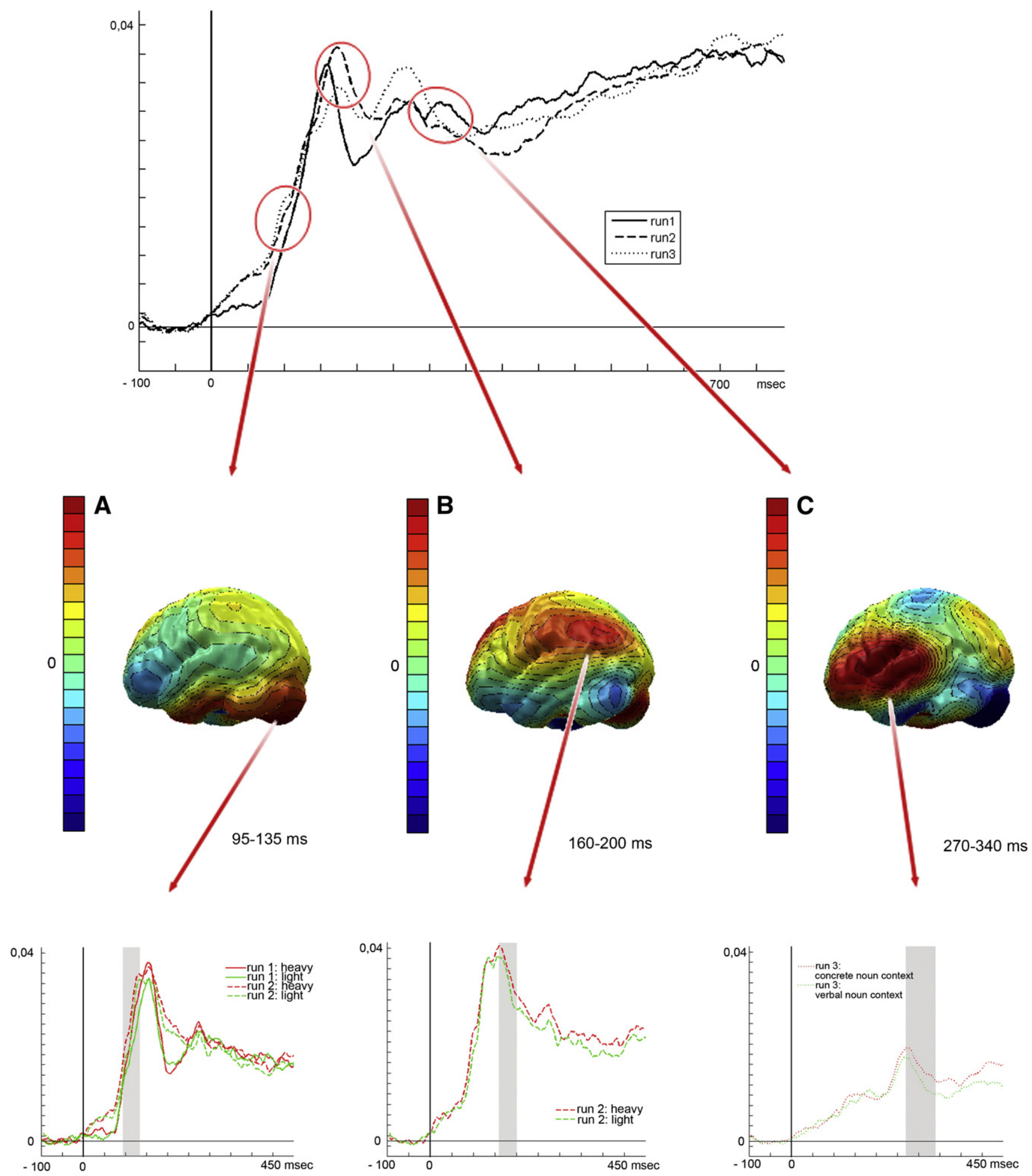
distinct brain response. Neurolinguistic studies on cortical correlates of German 'light' verb processing are rare compared to linguistic studies (Helbig and Buscha, 1999; Heringer, 1968; von Polenz, 1963; Winhart, 2002). Hence, it is difficult to derive specific hypotheses regarding the modulation of brain activity by the characteristic of 'light' versus 'heavy' verb reading. However, there are several possibilities to be explored. For one, following a Hebbian model of cortical neuronal networks reflecting the associative strength of lexical items (Pulvermüller, 1999), the underspecification of 'light' verbs might imply that they appear in a broader range of stimulus constellations and, hence, should activate widely distributed networks, whereas 'heavy' verbs activate more specific neuronal networks. Since the activation of distributed networks is usually reflected by smaller cortical responses, one would therefore expect less activation by 'light' in comparison to 'heavy' verbs. Alternatively, underspecification might provoke expectations regarding meaning. If those are not met, larger activity indicating deviation from expectancy might be observed in a similar manner to that of linguistic stimuli, which violate expected semantic or syntactic features and have been found to evoke a negative-going deflection in the evoked brain potential some $200 \mathrm{~ms}$ after stimulus onset (Friederici et al., 1993; Neville et al., 1991) for reviews (Friederici and Weissenborn, 2007; Pulvermüller, 1999). As yet another alternative, given the underspecification model of Lahiri and Reetz (2002), underspecification might instead prevent a violation of expectations, as a broader range of meanings should allow a 'no mismatch' rather than a 'mismatch' (Lahiri and Reetz, 2002). From this perspective, 'light' verbs should provoke less ambiguity and, hence, smaller cortical responses. Given the general success of Lahiri and Reetz's (2002) underspecification model in predicting cortical responses, we currently favor this hypothesis.

In the present study, conditions of verb presentation varied across three runs: verbs were presented (1) in isolation, or (2) in minimal context of a personal pronoun, in order to examine whether the specific characteristics of the verb category as underspecified or specific was carried by the verb itself or required a minimal context for distinct processing of its category features. In run (3), only 'light' verbs were presented in a full sentence context suggesting either a 'light' or 'heavy' use, in order to examine whether the ambiguity-resolving context would modify the cortical responses to 'light' verbs, that is, whether the meaning modified the response to one and the same morphological form. If so, sentence context resolving underspecification towards concrete ('heavy') meaning should modulate brain responses towards those evoked by 'heavy' verbs. Consequently, sentence context resolving underspecification towards abstract ('light') meaning should evoke brain responses similar to those evoked by 'light' verbs in the first two runs.

\section{Results}

Differences in the magnetic flux density between verb categories were evident between 100 and $500 \mathrm{~ms}$ after stimulus onset. The time course of the mean activity in the source space
(Fig. 1, top) disclosed three peaks of activity in response to verb onset, and topographical maps localized the time segments, in which activity distinguished 'heavy' and 'light' verbs in the three runs: in the first (verb in isolation, Fig. 1, mid A) and second run (verb in minimal context), responses differed around 95-130 ms. In the second run, additional differential activity occurred at 160-200 ms (Fig. 1, mid B). In the third run (verb in sentence context), activity distinguished 'light' verbs with different context-induced meaning between 270 and $340 \mathrm{~ms}$ after stimulus onset (Fig. 1, mid C). Except for the early peak around $100 \mathrm{~ms}$ with central occipital-parietal dominance (Hemisphere $\times$ Verb category, $F(1,21)=1.81, p>.1$ ), differential activation dominated in left hemispheric regions (160-200 ms: Hemisphere $\times$ Verb category, $F(1,21)=5.80, p<.05$; Hemisphere, $F(1,21)=3.70, p=.07 ; 270-340 \mathrm{~ms}$ : Hemisphere $\times$ Verb category $F(1,21)=184.26, p<.001$; Hemisphere, $F(1,21)=31.99, p<.01)$.

When presented in isolation and in minimal context, 'heavy' verbs evoked more pronounced posterior responses 95-135 ms after stimulus onset than 'light' verbs for a region of interest (ROI) defined as the average MNE amplitude of 6 dipoles in a central occipito-parietal region (Fig. 1, mid and bottom; Verb category, $F(1,21)=13.05, p<.01$; post hoc contrasts verified the significant differences for run $1, p<.01$ and run 2 , $p<.05$ ). In addition, verbs in minimal context evoked activity between 160 and $200 \mathrm{~ms}$, which was larger under this condition than in the other two runs (Run: $F(2,42)=4.20$, $p<.05)$. More pronounced activation by 'heavy' than by 'light' verbs was verified for a ROI in the left visual word form area (VWFA) defined by the average MNE over 6 dipoles (Fig. 1, mid and bottom; Verb category, $F(1,21)=4.36, p<.05)$.

When potential 'light' verbs were presented in concrete ('heavy reading') and verbal noun context ('light reading') in run 3, differential cortical responses were evident at 270$340 \mathrm{~ms}$ after stimulus onset in left-temporal regions: 'light' verbs in context signaling 'heavy reading' evoked larger responses than 'light' verbs in 'light reading' context for the ROI defined as average across 7 dipoles (Fig. 1, mid and bottom; Verb category, $F(1,21)=11.18, p<.01)$. Whereas the latter evoked similar activity as 'light' verbs presented in isolation or with minimal context (both post hoc comparisons across runs n.s.), 'light' verbs in 'heavy reading' context evoked even larger responses than 'heavy' verbs in runs 1 and $2(p<.01)$. A provisional (given the unbalanced stimulus categories) comparison across all three runs supported this results with the interaction Run $\times$ Verb category $(F(2,42)=3.23, p<.05)$.

For attention control, subjects performed a lexical decision task on real and pseudo verbs. Irrespective of runs, real verbs evoked more pronounced activity than pseudo verbs in left occipital regions around 110-150 ms (Hemisphere, $F(1,21)=$ 15.64, $p<.01$; Hemisphere $\times$ Verb category, $F(1,21)=153.92$, $p<.001)$. A main effect Verb category $(F(1,21)=22.34, p<.01$; post hoc contrasts: $p<.01$ in run 1 and $2, p<.05$ in run 3 ) verified this difference for a ROI defined by 8 dipoles in left occipital regions.

\section{Discussion}

Various linguistic and neurolinguistic (brain imaging) studies have addressed the representation and processing of 
semantic and syntactic features of lexical items. The present MEG study explored the processing of German function verbs defined as 'light' because of their potential ambiguous or underspecified semantic meaning in comparison to semantically unambiguous 'heavy' verbs. The spatio-temporal resolution of MEG data disclosed distinct cortical processing of morphologically identical verbs that were distinguished by this semantic feature. The early visually evoked response around $100 \mathrm{~ms}$ already varied with the verb category 'light' or 'heavy', suggesting rapid processing of the semantic distinction between ambiguous/underspecified or more specified meaning. The focus of this effect in central posterior brain areas suggests that the processing of such a lexical feature modulates early, preattentive stimulus processing in the visual cortex (corresponding to an event-related P100). Modulation of early visual processing by stimulus features has been documented for other stimulus features like the emotional valence of pictorial stimuli (Junghöfer et al., 2001) or emotional words (Bernat et al., 2001; Ortigue et al., 2004; Skrandies, 1998). As the effect was prominent already when verbs were presented without any context, information about the semantic features defining a verb as specified or underspecified seems to be represented in the verb itself. A similar distinct processing of isolated verbs differing in their argument structure information has been described, though at later latency (Assadollahi and Rockstroh, 2008).

Presentation of the verb in minimal context of a personal pronoun evoked the same early differential processing plus another differential activity at 160-200 ms. Activity in this latency range has been associated with processing of lexical features like verbs relative to nouns (Preissl et al., 1995, 2006), and content (noun, verb) relative to function words (Takashima et al., 2002).

The focus of the present differential activity in posterior regions, possibly the visual word form area, and its presence under stimulus presentation without syntactic violation argue against an interpretation as early left-anterior negativity (ELAN). As an alternative, it may be assumed that increasing complexity of processing (from single word to additional personal pronoun) requires more time for associative neuronal networks to become activated, which may then be reflected in later peaks of focal activity. This hypothesis is supported by the still later peak latency of activation under full sentence processing (run 3).

Whether presented in isolation or with minimal context, 'light' verbs evoked smaller activity than 'heavy' verbs. This is in line with the concept of underspecification of 'light' verbs: underspecification, which allows the integration of a broader range of meaning, should increase the probability of a nonmismatch (Lahiri and Reetz, 2002) and, hence, should evoke less 'mismatch-related' activation. This is also in line with a neuronal network model of lexical representations (Pulvermüller, 1999): as underspecified 'light' verbs may appear in a multitude of stimulus constellations, their representation in more extended cortical neuronal networks is to be expected. At the cortical surface average activity in extended and hence more distributed cortical neuronal networks may become evident in smaller response magnitude. As an alternative explanation, underspecified 'light' verbs may be supposed to have less features spelled out in neuronal substrate and thus activate less local neurons; Input variance is larger and correlation between features may therefore be lower. Again, a smaller focal neuronal network may be supposed to contribute to smaller response amplitude measured in locally constrained regions of interest. Which explanation holds depends on the inter-feature correlation, that is, how many features of the representations tend to correlate in how many linguistic situations. Pulvermüller (1999) based his model on evidence for function relative to content words. The present study adds evidence to extend the model to 'light' relative to 'heavy' verbs. Since the two verb categories were controlled for frequency, length, and number of arguments, an explanation of the distinct responses by one of these variables seems less likely.

Whenever 'light' verbs were presented in sentence context, the disambiguating context modulated left temporal activity around $300 \mathrm{~ms}$. This strengthens the hypothesis that disambiguating context changes the verb's feature despite of identical morphological form: context-induced 'heavy reading' specified a 'light' verb sufficiently to evoke similar responses as a specific 'heavy' verb.

Electromagnetic responses varied between real and pseudo verbs. Smaller response amplitudes to pseudo words relative to real words have been explained within the neuronal network model of lexical representations (Pulvermüller, 1999). The peak of distinguishing activity around $150 \mathrm{~ms}$ suggests to relate it to an early left anterior negativity (Friederici and Weissenborn, 2007). However, the posterior localization of the differential activation argues against an interpretation as ELAN, which is usually found in the inferior frontal cortex (IFG) or Broca's area (Friederici and Kotz, 2003). To what extent differences in experimental conditions (e.g., no syntactic violation) account for the different results remains to be proven. The present results are in line with other findings of left posterior activation distinguishing words and pseudo words between 100 and 150 ms and with activation distinguishing high- and low-frequent words in the VWFA between 150 and $200 \mathrm{~ms}$ (Assadollahi, unpublished data). It remains to be verified whether parieto-occipital activation indicates lexical access, and whether inferior-frontal activation indicates processing of syntactic violation.

Peak activity and effects of verb categories varied across verb presentation conditions. Peak latencies may be assumed to increase with the number of stimuli that add up to form a meaningful sentence, but the rapid processing of semantic and syntactic stimulus features seems independent of this number and may be reactivated, when minimal or sentence context are to be processed. The present results of the spatiotemporal images show the complex relationship between each single complement of sentence, their interaction, the different levels of information, and nevertheless any lack of information (underspecified 'light' verbs). The processing of single words, phrases (as next larger unit), and whole sentences (as complete denoted events) is hierarchical. Any new incoming information during reading is built up on preceding and further information, independent of sentence type. That is, thematic roles correspond to grammatical functions (subject, object), and those relate to expressions of case (nominative, accusative, etc.). Information depends on each other to advance sentence meaning. In case of verb 
parsing, the processor first 'encodes' the features carried by the verb, which 'triggers' the type of the participants taking part in the described event. Next, the processor then 'decodes' the information thus obtained into expressions of case and abstract concepts of grammatical functions, which defines the syntax-semantic interface, the chronological mapping of the syntactic argument structure onto the semantic argument list of a verb (thematic roles). This process may have become evident in the present activity, which reflects properties of participants. Activity in left temporal regions increases when expressions of case and grammatical functions are mapped onto the thematic roles. In simple ('heavy' verb) sentences parsing is successful as all participants are semantically defined and the 'decoding' can take place. In complex predicates, however, the linear order of the single component parts differs. Sentence parsing regarding complex predicates involves the verbal noun for assigning thematic roles. Thereby, the argument-merging supposed to be manifest as both parts of the predication license the argument structure of one single event. This may have become evident in the activity peak around $300 \mathrm{~ms}$ in sentence context.

The spatio-temporal sequence of effects suggests that specific neuronal networks are involved in the lexical item processing: distinction of real and pseudo words requires early (100-150 ms) activity in left occipital regions, the processing of a verb presented in minimal context activates the left VWFA around $200 \mathrm{~ms}$, and resolution of semantic meaning by disambiguating context activates left temporal regions at even longer latency around $300 \mathrm{~ms}$.

In sum, the present results indicate distinct cortical processing of verbs that are distinguished by their semantic reading as 'light' or 'heavy'. Whether or not they are presented within a context that resolves the ambiguity of meaning. The smaller activation by 'light' verbs supports interpretation of 'light' as underspecified. The present study strengthens the linguistic concept that the meaning of a verb is represented as a lexical event structure - the participants of the described event. Moreover, the combination of a verb with other lexemes is subject to semantic restrictions.

\section{Experimental procedures}

\subsection{Subjects}

Thirty-eight native German-speaking subjects (19 males; mean age \pm SD $24.4 \pm 3.1$ years) were recruited. All subjects were normal-sighted and right-handed. Data of 16 subjects had to be excluded from data analyses because of an inadequate percentage of correct responses or insufficient MEG data quality in any one of the three conditions. Thus, data of 22 subjects ( 13 males, mean age \pm SD $24.6 \pm 3.5$ years) entered present analyses.

\subsection{Stimulus material}

Stimuli were selected in a pilot study: thirty-four native German speakers evaluated the degree of ambiguity of 54 verbs on an 8-point Likert scale ( 8 =ambiguous, $1=$ unambiguous). The sequence of stimuli varied from subject to subject in order to control for a sequence effect. As a result of the ratings, 20 verbs were selected as 'heavy' (unambiguous; mean rating 4.94) and 20 verbs as 'light' (ambiguous; mean rating 2.98; $\left.\mathrm{t}_{(19)}=-12.17, p<.01\right)$. The two stimulus sets were matched for word frequency $\left(t_{(38)}=-1.5, p=n\right.$.s.) and valence $\left(t_{(38)}=-1.0, p=n\right.$.s.; Schulte im Walde, 2002). An additional set of 50 orthographically legal but meaningless pseudo verbs were chosen for the lexical decision task. The three verb categories did not differ in word length in characters $(F(2,38)=$ 1.97, $p=$ n.s.).

For the presentation of 'light' verbs in sentence context, 20 concrete nouns and 20 abstract (verbal) nouns were chosen, which determined the verb's 'heavy' versus 'light reading'. All noun phrases fitted the most frequent category use for the respective verb (Schulte im Walde, 2002). Sentences with 'heavy' and 'light reading' context consisted of equal number of words (transitive sentence with 3 words each), equal category of complement (noun phrase accusative: $n=14$, prepositional phrase: $n=5$, noun phrase accusative + prepositional: $n=1$ for 'heavy' and 'light reading' each), and equal German personal pronoun use ("er"/"he": $n=9$, "sie"/ "she": $n=9$, "es"/“it": $n=2$, for 'heavy' and 'light reading' each). A full sentence context was also constructed for each of the 50 pseudo verbs. All sentences were constructed in identical morphological form: active and simple present tense ("Einen Entschluss fasst er"/“A decision he takes").

Each of the three runs comprised 250 stimulus presentations, with 'heavy' and 'light' verbs (sentences with 'light' verbs of either 'heavy' or 'light reading') being repeated five times each, but pseudo verbs (respective pseudo verb sentences) only once within each run. Within each run, stimulus presentation followed a pseudo random order. Stimulus presentation was realized via video beamer (JVCTM, DLAG11E) and a mirror system approximately $80 \mathrm{~cm}$ in front of the subjects. Words consisted of white letters against a dark background.

\subsection{Design and procedure}

The experimental protocol was approved by the ethical committee of the University of Konstanz. Subjects were informed about experimental and measurement procedures and signed a written informed consent. At the end of the experimental session, subjects received a financial bonus.

All subjects were instructed to silently read each verb and to indicate by button press, whether the presented verb was a real verb or a pseudo verb. The association of left/right button and real/pseudo verb was balanced across subjects. The lexical decision was followed by an inter-stimulus interval (ISI) that varied randomly between 1200 and $1800 \mathrm{~ms}$.

The experimental session comprised three runs. In the first run, verbs were presented in isolation. In the second run, each verb was preceded by a personal pronoun (minimal context). The third run comprised only 'light' verbs, which were presented in either concrete ('heavy reading') or verbal noun ('light reading') context. Full sentences were presented with the verb preceded by the context and followed by a personal pronoun.

Each visual stimulus (verb, personal pronoun, and context noun) was presented for $150 \mathrm{~ms}$. Presentation of the personal 
pronoun and the verb in run 2 as well as noun, verb, and personal pronoun in run 3 were separated by a betweenstimulus interval varying between 400 and $600 \mathrm{~ms}$.

\subsection{Data acquisition and analysis}

Electromagnetic signals were recorded with a 148-channel magnetometer (Magnes 2500 WH, BTi, San Diego, CA) using a 0.1-100 $\mathrm{Hz}$ band-pass filter and a sampling rate of $678.17 \mathrm{~Hz}$. Vertical and horizontal eye movements (electrooculogram, EOG) were recorded for artifact control. After external global noise subtraction, continuous MEG data were split into $900 \mathrm{~ms}$ epochs excluding artifact-contaminated epochs (EOG level $>100 \mu \mathrm{V}$, MEG level $>5 \mathrm{pT}$, button press). A trigger marked the onset of each verb stimulus for trial extraction from the continuous MEG data. For trials with correct responses, stimulus-locked evoked magnetic fields (EMFs) were determined relative to a $100 \mathrm{~ms}$ pre-stimulus baseline interval. An average of $75 \%$ of trials was accepted per condition and subject.

Cortical sources of activity were determined from the average EMFs using the Minimum Norm Estimate (MNE) based on a spherical volume conductor (Hauk et al., 2002). The MNE (Hämäläinen et al., 1993; Hämäläinen and Ilmoniemi, 1994) represents an objective inverse method to reconstruct the topography of the primary current underlying a magnetic field distribution within acceptable residual variance (here: $<5 \%$ ). Pseudo-inversion matrices were regularized (Tikhonov-Phillips, $\lambda=0.01$ ). Cortical activity was estimated in a threedimensional source space consisting of four concentric spherical shells, with the outer shell being fitted to the individual headshape of the subjects (4D Neuroimaging software). According to Sarvas (1987), the radius of the head has no effect on the estimated magnetic field generated by primary currents in a spherically symmetric volume conductor. For the present analysis, the head radius was estimated to be $10 \mathrm{~cm}$. MNE are reported for the shell at $80 \%$ of this radius which roughly corresponds to the cortex in the brain. On this sphere, 197 dipole pairs were arranged uniformly on a geodesic net. Each pair consisted of two orthogonal dipoles. Both dipoles were oriented tangentially to the surface, and dipoles in relation to each other were orthogonal to capture " $x$ " and " $y$ " components at the surface. The RMS amplitude of each pair was used. MNE magnitudes were averaged for each subject and verb category.

Experimental effects on the spatio-temporal activity pattern were evaluated in a step-wise procedure: (1) the global mean (Fig. 1, top) illustrates activity peaks around $100 \mathrm{~ms}$, $200 \mathrm{~ms}$ and $300 \mathrm{~ms}$ after stimulus onset; (2) topographical maps (Fig. 1, mid) curtailed time segments in which activity distinguished verb categories in the three runs at 95-135 ms, 160-200 ms, and 270-340 ms after stimulus onset. For these time segments, lateralization was first evaluated by repeatedmeasures analyses of variance (ANOVA) comparing average left- and right-hemispheric MNE by the within-subject factor Hemisphere; (3) for time segments with significant interaction Hemisphere $\times$ Verb category, indicating dominance of differential cortical responses to verb categories in the left hemisphere, further analyses were confined to left hemispheric ROI. For these ROI, differences in average MNE amplitudes between verb categories were verified by repeated measures ANOVA with the within-subjects factors Verb category (comparing responses to 'heavy' and 'light' verbs in run 1 and 2, 'light' verbs in concrete-versus verbal-noun-context in run 3 , or verbs versus pseudo verbs across runs). An additional ANOVA compared effect of verbs presented in isolation versus in context with the within-subject factor Run. Another exploratory ANOVA included all three runs, although strict comparison was not justified because of the unequal distribution of stimulus types ('light' verbs only in run 3). Significant main effects were gradually decomposed using Fisher LSD test.

\section{Conflict of interests}

The authors declare that they have no financial or nonfinancial competing interests.

\section{Acknowledgment}

Research was supported by the German Research Foundation (DFG, Collaborative Center Grant 471).

\section{REFEREN C E S}

Assadollahi, R., Pulvermüller, F., 2003. Early influences of word length and frequency: a group study in the MEG. NeuroReport 14 (8), 1183-1187.

Assadollahi, R., Rockstroh, B., 2008. Representation of the verb's argument-structure in the human brain. BMC Neurosci. 9, 69.

Bernat, E., Bunce, S., Shevrin, H., 2001. Event-related brain potentials differentiate positive and negative mood adjectives during both supraliminal and subliminal visual processing. Int. J. Psychophysiol. 42 (1), 11-34.

Bresnan, J., Zaenen, A., 1990. Deep unaccusativity in LFG. In: Dziwirek, K., Farrell, P., Mejías-Bikandi, E. (Eds.), Grammatical Relations. A Cross-Theoretical Perspective. CSLI Publications, Stanford, CA, pp. 45-57.

Butt, M., 1995. The Structure of Complex Predicates in Urdu. CSLI Publications, Stanford, CA.

Butt, M., 2003. The light verb jungle. Harvard Working Papers in Linguistics 9, 1-49.

Butt, M., Geuder, W., 2001. On the (Semi) Lexical status of light verbs. In: Corver, N., van Riemsdijk, H.C. (Eds.), Semi-lexical Categories: The Function of Content Words and the Content of Function Words. Walter de Gruyter, Berlin, pp. 323-370.

Butt, M., Tantos, A. 2004. Verbal Semantics via Petri Nets, Paper presented at the LFG04 Conference.

Butt, M., Lahiri, A. 2007. Historical Stability vs. Historical Change. Konstanz, 2002. http://ling.uni-konstanz.de/pages/home/butt/ stability.pdf.

Butt, M., Dalrymple, M., A., F., 1997. An architecture for linking theory in LFG. In: Butt, M., King, H.T. (Eds.), Proceedings of the LFG97 Conference. University of California, San Diego. http:// csli-publications.stanford.edu/LFG/2/lfg97.html.

Butt, M., King, H.T., Ramchand, G., 2008. Complex predication: who made the child pinch the elephant? In: Uyechi, L., Wee, L.H. (Eds.), Reality Exploration and Discovery Pattern Interaction in Language and Life. CSLI Publications.

Dalrymple, M., 2001. Lexical Functional Grammar, Syntax and Semantics Series, Vol. 42. Academic Press, New York.

Friederici, A.D., Kotz, S.A., 2003. The brain basis of syntactic processes: functional imaging and lesion studies. Neuroimage 20, 8-17. 
Friederici, A.D., Weissenborn, J., 2007. Mapping sentence form onto meaning: the syntax-semantic interface. Brain Res 1146, 50-58.

Friederici, A.D., Pfeiffer, E., Hahne, A., 1993. Event-related brain potentials during natural speech processing: effects of semantic, morphological and syntactic violations. Brain Res. Cogn. Brain Res. 1 (3), 183-192.

Grimshaw, J., Mester, A., 1988. Light verbs and theta-marking. Linguist. Inq. 19 (2), 205-232.

Hämäläinen, M., Ilmoniemi, R.J., 1994. Interpreting magnetic fields of the brain: minimum norm estimates. Med. Biol. Eng. Comput. 32 (1), 35-42.

Hämäläinen, M., Hari, R., Ilmoniemi, R.J., Knuutila, J., Lounasmaa, O.V., 1993. Magnetoencephalography - theory, instrumentation, and applications to noninvasive studies of the working human brain. Rev. Mod. Phys. 65 (2), 413-497.

Hauk, O., Keil, A., Elbert, T., Müller, M.M., 2002. Comparison of data transformation procedures to enhance topographical accuracy in time-series analysis of the human EEG. J. Neurosci. Res. 113, 111-122.

Helbig, G., Buscha, J., 1999. Deutsche Grammatik. Ein Handbuch für den Ausländerunterricht. 19. Auflage ed. Langenscheidt, Verlag Enzyklopädie, Leipzig.

Heringer, H.J., 1968. Die Opposition von 'kommen' und 'bringen' als Funktionsverben. Untersuchungen zur grammatischen Wertigkeit und Aktionsart. Schwann. Düsseldorf.

Higginbotham, J., 1983. The logic of perceptual reports: an extensional alternative to situation semantics. J. Philos. 80, 100-127.

Junghöfer, M., Peyk, P., 2004. Analyse und Visualisierung von Hirnstrom-und Hirnmagnetfeld-Messungen. Matlab. Select. 2, 24-28.

Junghöfer, M., Bradley, M.M., Elbert, T., Lang, P.J., 2001. Fleeting images: a new look at early emotion discrimination Psychophysiology 38, 175-178.

Lahiri, A., Reetz, H., 2002. Underspecified recognition. In: Gussenhoven, C., Werner, N. (Eds.), Laboratory Phonology VII. Mouton, Berlin, pp. 637-675.
Neville, H.J., Nicol, J.L., Barss, A., 1991. Syntactically based sentence processing classes: evidence from event-related brain potentials. J. Cogn. Neurosci. 3, 151-165.

Ortigue, S., Michel, C.M., Murray, M.M., Mohr, C., Carbonnel, S., Landis, T., 2004. Electrical neuroimaging reveals early generator modulation to emotional words. Neuroimage 21 (4), 1242-1251.

Preissl, H., Pulvermüller, F., Lutzenberger, W., Birbaumer, N., 1995. Evoked potentials distinguish between nouns and verbs. Neurosci. Lett. 197, 81-83.

Preissl, H., Pulvermüller, F., Lutzenberger, W., Birbaumer, N., 2006. Brain rhythms of language: nouns versus verbs. Eur. J. Neurosci. 8, 937-941.

Pulvermüller, F., 1999. Words in the brain's language. Behav. Brain Sci. 22, 253-336.

Pulvermüller, F., Härle, M., Hummel, F., 2001. Walking or talking?: behavioral and neurophysiological correlates of action verb processing. Brain Lang. 78, 143-168.

Sarvas, J., 1987. Basic mathematical and electromagnetic concepts of the biomagnetic inverse problem. Phys. Med. Biol. 32 (1), 11-22.

Schulte im Walde, S., 2002. A Subcategorisation Lexicon for German Verbs Induced from a Lexicalised PCFG. Stuttgart.

Skrandies, W., 1998. Evoked potential correlates of semantic meaning - a brain mapping study. Brain Res. Cogn. Brain Res. 6 (3), 173-183.

Takashima, A., Ohta, K., Shirahama, Y., Matsushima, E., Horiguchi, T., Nishikawa, T., 2002. Difference of semantic processing and character types in silent reading of words-a study using MEG. Int. Congr. Ser. 1232, 513-517.

von Polenz, P., 1963. Funktionsverben im heutigen Deutsch. Sprache in der rationalisierten Welt. Schwann. Düsseldorf.

Winhart, H., 2002. Funktionsverbgefüge im Deutschen. Zur Verbindung von Verben und Nominalisierungen, Neuphilologische Fakultät, Vol. Philosophische Dissertation. Universität Tübingen, Tübingen. http://w210.ub.uni-tuebingen. de/dbt/volltexte/2005/1696/. 